Article

\title{
In-Orbit Operational Parameter Calculation and Performance Optimization in KOMPSAT-6 Synthetic Aperture Radar
}

\author{
Jin-Bong Sung ${ }^{1,2}$ and Sung-Yong Hong ${ }^{1, *}$ \\ 1 Department of Radio and Information Communications Engineering, Chungnam National University, \\ Daejeon 305-764, Korea; courtneys@o.cnu.ac.kr \\ 2 Agency for Defense Development, Daejeon 34186, Korea \\ * Correspondence: syhong@cnu.ac.kr; Tel.: +82-428216885
}

check for updates

Citation: Sung, J.-B.; Hong, S.-Y. In-Orbit Operational Parameter Calculation and Performance Optimization in KOMPSAT-6 Synthetic Aperture Radar. Remote Sens. 2021, 13, 2342. https:// doi.org $/ 10.3390 /$ rs13122342

Academic Editor: Timo Balz

Received: 15 May 2021

Accepted: 12 June 2021

Published: 15 June 2021

Publisher's Note: MDPI stays neutral with regard to jurisdictional claims in published maps and institutional affiliations.

Copyright: (c) 2021 by the authors. Licensee MDPI, Basel, Switzerland. This article is an open access article distributed under the terms and conditions of the Creative Commons Attribution (CC BY) license (https:/ / creativecommons.org/licenses/by/ $4.0 /)$.

\begin{abstract}
A new method to design in-orbit synthetic aperture radar operational parameters has been implemented for the Korean Multi-purpose Satellite 6 mission. The implemented method optimizes the pulse repetition frequency when a satellite altitude changes from its nominal one, so it has the advantage that the synthetic aperture radar performances can satisfy the requirements for the in-orbit operation. Other commanding parameters have been designed to conduct trade-off between those parameters. This paper presents the new optimization method to maintain the synthetic aperture radar performances even in the case of an altitude variation. Design methodologies to determine operational parameters, respectively, at nominal altitude and in orbit are presented. In addition, numerical simulation is presented to validate the proposed optimization and the design methodologies.
\end{abstract}

Keywords: range ambiguity improvement; azimuth ambiguity improvement; noise equivalent sigma nougat improvement; PRF selection

\section{Introduction}

This paper proposes a method to design the synthetic aperture radar (SAR) operational parameters for the Korean Multi-purpose Satellite (KOMPSAT) 6. Among those parameters, pulse repetition frequency (PRF) is an important one because it determines the ambiguity ratios including the range ambiguity ratio (RAR) and azimuth ambiguity ratio (AAR). PRF is determined using a nominal altitude but should be optimized as the in-orbit altitude changes from the nominal altitude. The proposed method uses the traveling return time to optimize PRF. Utilization of the traveling return time enables PRF against the altitude variation to be corrected, and consequently, the altitude variation does not degrade the SAR performances including the PRF and slant range margins. In addition, the proposed method does not cause any overload for the SAR controller, so the on-board optimization for PRF is possible to realize a real-time operation of a SAR satellite. Instead of the incidence angle, the proposed method uses the look angle for the reference angle to improve the SAR performances. Numerical simulation shows that the proposed method achieves higher SAR performances even for the altitude variation in comparison with the previous methods, which include the one used for KOMPSAT-5.

As the first SAR satellite in Korea, KOMPSAT-5 has been launched successfully in August of 2013 [1-4]. To enhance mission capability and achieve a new operational concept, an active phased array SAR payload is being developed for KOMPSAT-6. The SAR payload has features of high flexibility in functional architecture, enhanced performances, and new operational modes such as terrain observation progressive scan (TOPS) [5,6]. Considering the osculating orbit shown in Figure 1, a satellite altitude on the World Geodetic System (WGS) 84 is dependent on both the orbit eccentricity and earth flatness. For one cycle, days and the number of orbits are 11 and 167, respectively, so the mean altitude is $505.373 \mathrm{~km}$. Figure 1 shows the predicted satellite altitude along the elapsed time from 1 Jan. 2022. Each point in Figure 1 represents the satellite altitude that is predicted at every 5 min for a specific 
day. The darker color between 509 and $522 \mathrm{~km}$ means that the satellite is located over that altitude range more frequently over $522 \mathrm{~km}$. On the WGS84 ellipsoid, the whole variation in altitude is about $30 \mathrm{~km}$ for the orbit eccentricity of 0.002 . For the in-orbit operation, the operational parameters are calculated at the ground segment using the nominal altitude. The calculated parameters are then used to operate SAR. When the satellite has an altitude different from the nominal one, the ground segment uses the tables on the nominal swath and macro-command parameter, which are stored in database, to recalculate the actual in-orbit parameters at a new altitude that is predicted by the ground segment [7]. The SAR operational parameters are optimized based on the predicted altitude at the ground segment. Thus, the SAR performance and image quality strongly depend on accuracy of the optimized SAR operational parameter. The SAR operational parameters are generated using a SAR design and analysis tool to meet the SAR performance requirement [6]. The generation process calculates radar instrument parameters like PRF, the echo window timing, receiver gain settings, the azimuth antenna steering sequence. Such generation process is repeated for each scene on the ground to be imaged. The SAR performance parameters are traded off for the number of iterations to meet the SAR requirement.

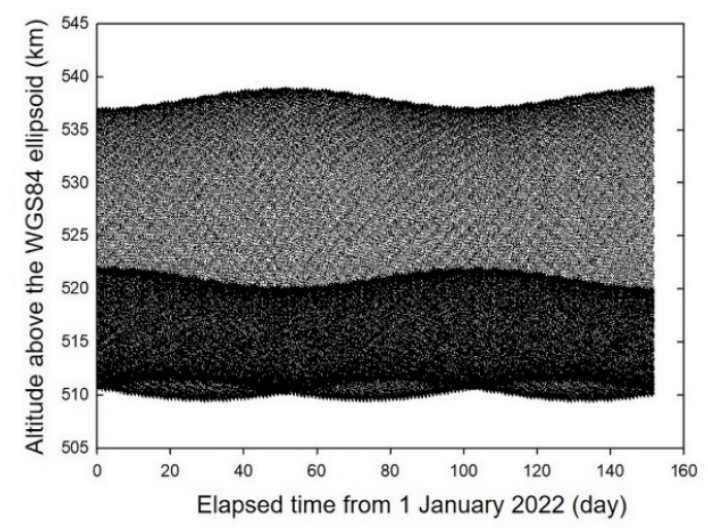

Figure 1. Satellite altitude predicted on the WGS84.

In this paper, a novel calculation method for the in-orbit SAR operational parameters is proposed. As before, the operational parameters are calculated using the nominal satellite altitude at the ground segment and then constructed as a database format. This paper proposes a new formula using PRF to calculate the actual parameter for the predicted altitude. Simulation results show that the proposed method improves the SAR performance even for altitude variation. This paper also investigates a reference angle. Numerical simulation for the swath design and the parameter optimization validates the effectiveness of the proposed method. This paper is organized as follows. Section 2 proposes design methodology to obtain the SAR operational parameter at the nominal satellite altitude. Section 3 proposes an optimization method to improve the SAR operational parameters on the condition of the altitude variation and performance measures that are expected from the previous heritage. Finally, Section 4 concludes this paper.

\section{Designing Nominal SAR Operational Parameters at Nominal Altitude}

Figure 2 shows a general design flow including an iteration process for SAR. The design flow starts from defining basic specifications, such as resolution, incident angle range, swath width, nominal orbit altitude, and so on. Available platform resources such as power consumption imposes another constraint to a mission design. Mission requirements are also used to define the SAR operational parameters such as Tx bandwidth, Tx duty cycle, azimuth integration time, acquisition time, pulse length, PRF and so on. As shown in Figure 2, the iteration process is used to determine PRF satisfying the requirements. 


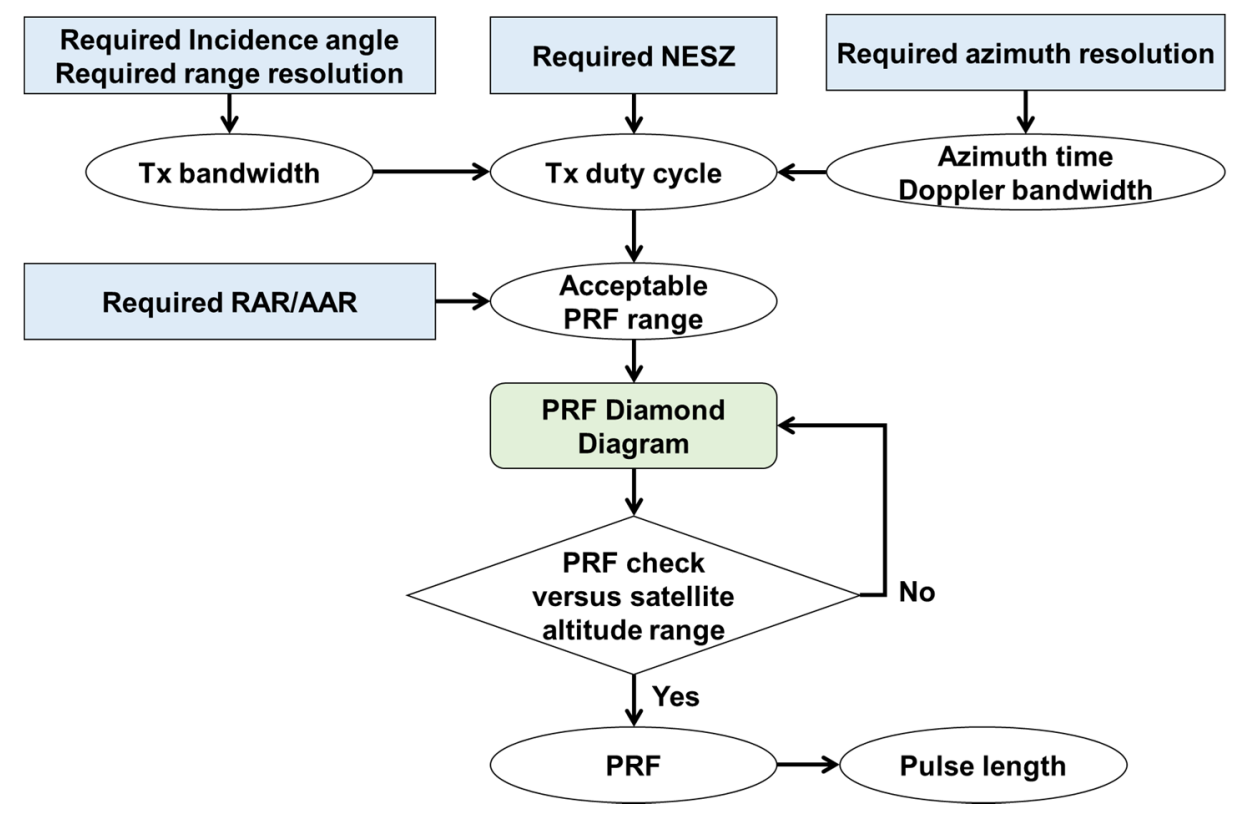

Figure 2. General SAR design flow and iteration process.

\subsection{Mission Requirement Definition}

Table 1 shows the mission requirements including the satellite altitude calculated using orbit repetition and eccentricity. The altitude variation is about $30 \mathrm{~km}$. The required ground swath width is $5 \mathrm{~km}$ for the sliding spotlight mode. The noise equivalent sigma zero (NESZ), range ambiguity ratio (RAR) and azimuth ambiguity ratio (AAR) are required to be less than or equal to $-20 \mathrm{~dB}$, respectively, within incidence angle range between 20 and $55^{\circ}$.

Table 1. Mission requirement for sliding spotlight mode.

\begin{tabular}{cc}
\hline Parameters & Value \\
\hline Satellite altitude & $509-540 \mathrm{~km}$ \\
Incidence angle range & $20-55^{\circ}$ \\
Ground swath width & $\geq 5 \mathrm{~km}$ \\
NESZ & $\leq-20 \mathrm{~dB}$ \\
RAR & $\leq-20 \mathrm{~dB}$ \\
AAR & $\leq-20 \mathrm{~dB}$ \\
\hline
\end{tabular}

\subsection{PRF Selection Considering Ambiguity}

PRF should be determined considering its impact on RAR and AAR, whose values are presented in Table 1. The RAR and AAR are calculated according to (1) and (2), respectively $[8,9]$.

$$
\begin{gathered}
\text { RAR }=\frac{\sum_{n \neq 0} G^{2}\left[\theta\left(\tau+\frac{n}{P R F}\right)\right] \frac{\sigma_{0}\left(\psi_{\mathrm{a}}\right)}{R_{\mathrm{a}}^{3} \sin \psi_{\mathrm{a}}}}{G^{2}[\theta(\tau)] \frac{\sigma_{0}\left(\psi_{\mathrm{t}}\right)}{R_{\mathrm{t}}^{3} \sin \psi_{\mathrm{t}}}} \\
\mathrm{AAR}=\frac{\sum_{m \neq 0} \int_{-\frac{B_{\mathrm{D}}}{2}}^{+\frac{B_{\mathrm{D}}}{2}} G^{2}(f+m \cdot P R F) d f}{\int_{-\frac{B_{\mathrm{D}}}{2}}^{+\frac{B_{\mathrm{D}}}{2}} G^{2}(f) d f}
\end{gathered}
$$

Table 2 shows symbols in Equations (1)-(2) and description of each symbol. 
Table 2. Symbols and description for (1) and (2).

\begin{tabular}{cc}
\hline Symbols & Description \\
\hline$\tau$ & Echo delay time projected into the cross-track ground plane \\
$\theta$ & Elevation angle \\
$\varphi$ & Azimuth angle \\
$G^{2}$ & Two-way far field antenna power pattern \\
$\sigma_{0}$ & Backscattering coefficient \\
$\psi_{\mathrm{t}}$ & Incidence angle from target signal \\
$\psi_{\mathrm{a}}$ & Incidence angle from ambiguous signal \\
$R_{\mathrm{t}}$ & Slant range of target signal \\
$R_{\mathrm{a}}$ & Slant range of ambiguous signal \\
$B_{\mathrm{D}}$ & Target Doppler bandwidth \\
\hline
\end{tabular}

Figure 3 shows the ambiguity-to-signal ratio (ASR) for an exemplary swath \#5. Figure 3 a shows positions where the RAR and AAR performances are investigated while Figure $3 \mathrm{~b}$ shows the RAR and AAR performances at each position. The RAR and AAR performances are better at the center than at the edges. RAR is degraded at higher PRF, but AAR has sinusoidal-like dependency for PRF. The AAR performance shows different trends along the scene in the azimuth direction. Usually, the performance at the edge of one scene is worse than one at the center of that scene. This is because the grating lobes are raised due to an azimuth beam steering in the sliding spotlight mode. PRF should be chosen to minimize the RAR and AAR performances in both azimuth and range direction. As shown in Figure 3, the best PRF range is about $9 \mathrm{kHz}$ between the dotted lines in grey.

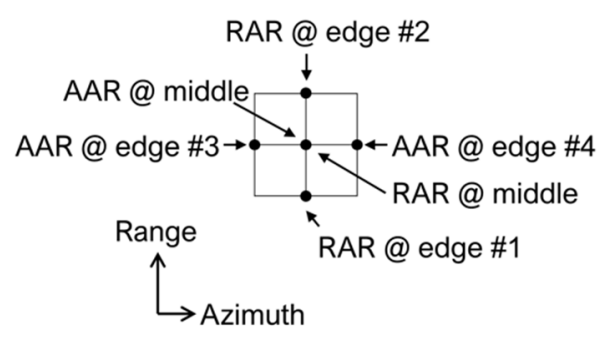

(a)

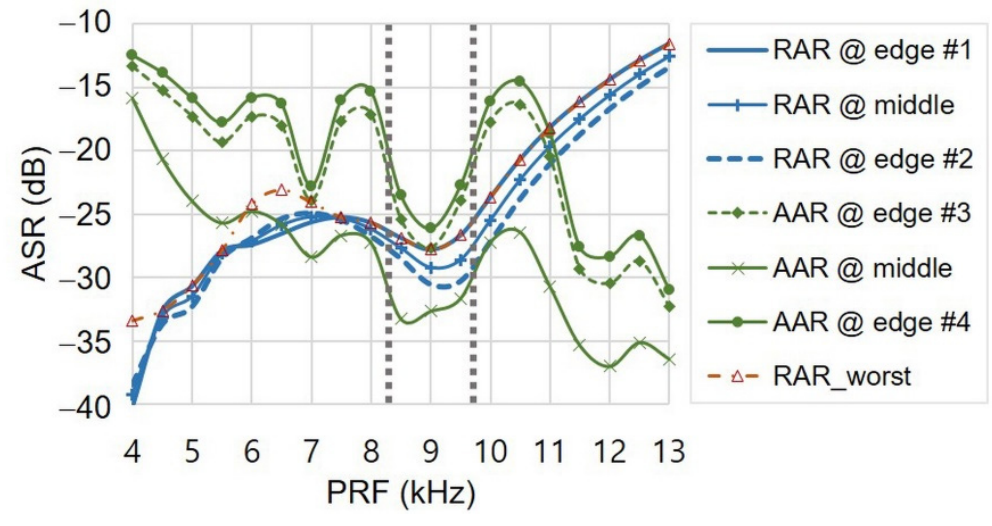

(b)

Figure 3. RAR and AAR performances for PRF: (a) position of RAR and AAR performances; (b) exemplary ASR performance in SW\#5.

Important factors in PRF selection are the echo timing constraints and ASR. To achieve the incidence angle from 20 to $55^{\circ}$, a total of 115 different swaths are assigned for the sliding spotlight mode. Instead of showing all the assigned swaths, only eight swaths are selected to present in this paper. The selected swaths have angle space of $5^{\circ}$ between the adjacent swaths. For simulation, the satellite altitude, swath width, and Tx duty ratio are set to $520 \mathrm{~km}, 5.3 \mathrm{~km}$, and 30\%, respectively. Figure 4 shows the simulated RAR performances for each of the selected swaths. The RAR values correspond to the worst ones shown in Figure $3 \mathrm{~b}$. For all the swaths, the RAR performance becomes better for the increasing PRF when PRF is larger than a specific value. In order to satisfy -20-dB RAR in Table 1 , the PRF range should be decreased for the increasing incidence angle, i.e., the increasing swath number SW\#. 


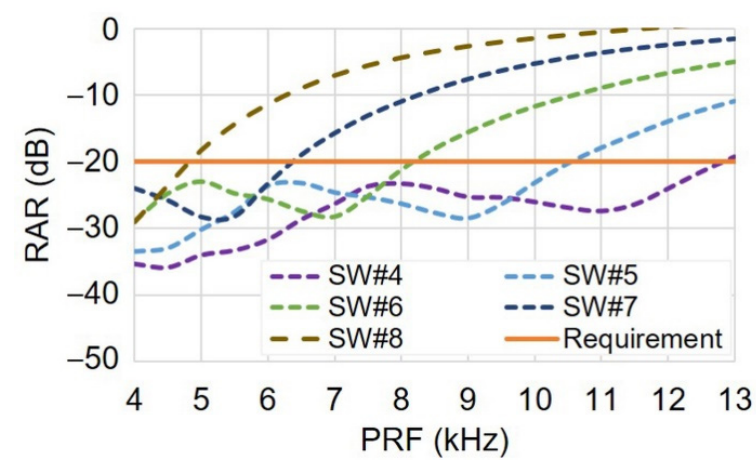

Figure 4. RAR performances for PRF.

Figure 5 shows the simulated AAR performances. The AAR performances are the same for all the swaths, so AAR can be easily estimated for all the swath numbers, respectively, as long as AAR is known for one swath. Such independency of AAR on the swath number is clear from (2) because the Doppler bandwidth, $B_{\mathrm{D}}$, is almost constant for all the swaths.

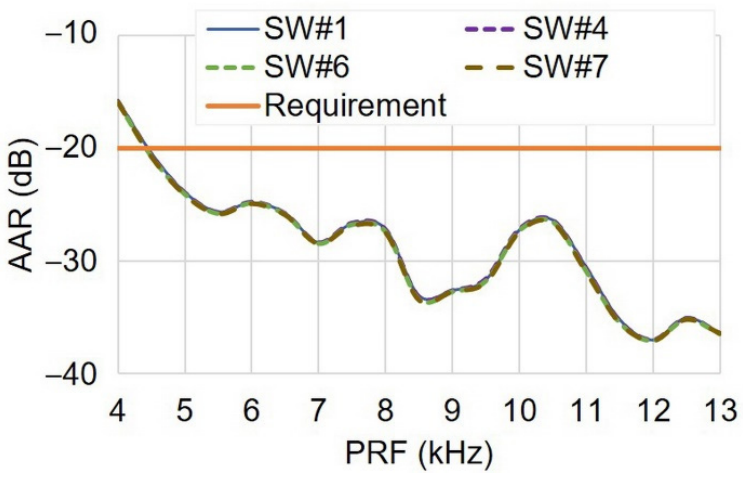

Figure 5. AAR performances for PRF.

The RAR and AAR performances within access region are shown in Figure 6, in which the RAR and AAR performances of Figures 5 and 6 are overlapped in a single figure. PRF should be selected from Figure 6 to satisfy -20-dB ASR for both RAR and AAR performances. In Figure 6, the dotted line in red represents PRF around $4.6 \mathrm{kHz}$ that is optimum value for PRF because it satisfies the required RAR and AAR performances for both range and azimuth directions as well as for the all the access regions from 20 to $55^{\circ}$. In this way, the optimum PRF can be effectively selected while saving time required for the optimization procedure because the reasonable PRF is intuitively selected among many swaths. Such PRF selection is verified by checking the PRF diamond diagram, which is described in the following section.

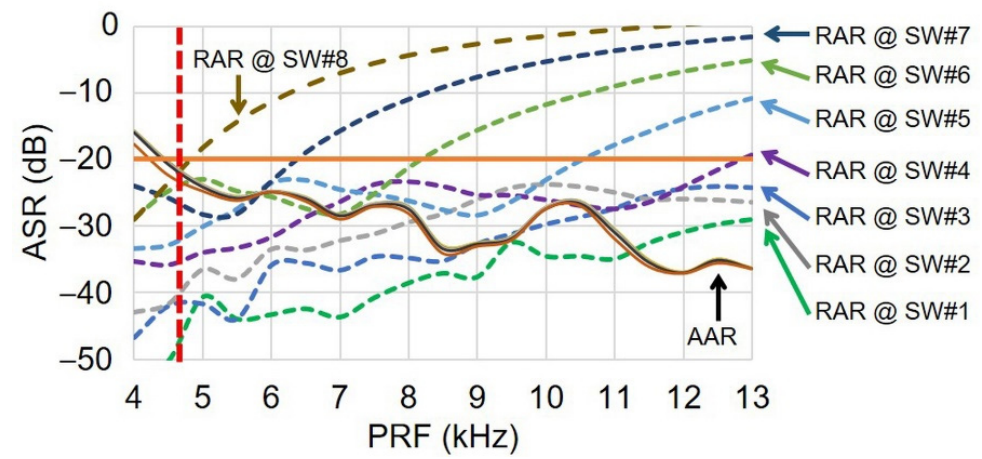

Figure 6. PRF selection from RAR and AAR performances. 


\subsection{PRF Selection and Timing}

A swath should be allocated in such a way that each pulse repetition interval (PRI) is wide enough to receive all echoes returning from the swath on the ground. For the $R x$ sampling, the total window length should be wider than the sum of all the differences of the near and far slant ranges with the Rx chirp duration, which is equal to the Tx chirp duration. As shown in Figure 7, this criterion is used to determine the allocable area for the Rx sampling after setting PRI and Tx pulse duration, $\tau_{\mathrm{p}}$, respectively. Rank in Figure 7 means the number of pulses within the delay time that is experienced by echo signal from the near slant range.

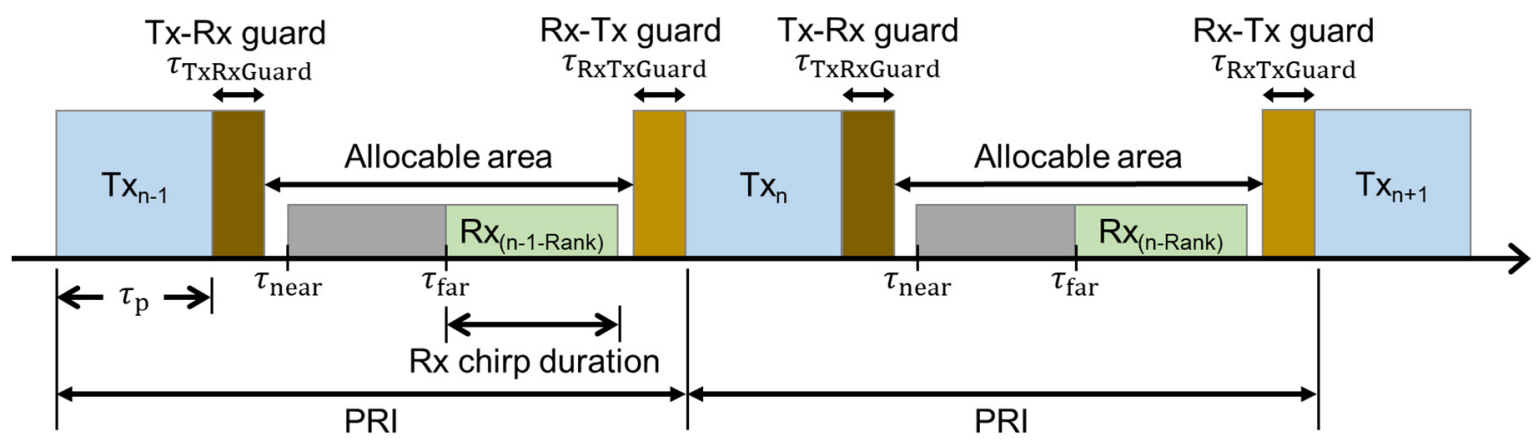

Figure 7. Allocable swath area.

Like in a general radar system, the SAR receiver is in a blind stage during the transmitting event due to weak isolation between the transmitter and receiver. When the returning echo coincides with the transmitting event, the received signal is eclipsed. PRF should be set to avoid the eclipsing. For this, PRF must satisfy the following inequality.

$$
\frac{N-1}{\tau_{\text {near }}-\tau_{\mathrm{p}}-\tau_{\text {TxRxGuard }}}<P R F<\frac{N}{\tau_{\text {far }}+\tau_{\mathrm{p}}+\tau_{\text {RxTxGuard }}}(N=1,2,3, \ldots)
$$

As shown in Figure $7, \tau_{\text {near }}$ and $\tau_{\text {far }}$ represent the respective return time for the slant range, which is defined as a time interval between the near and far edges in one swath. Also, $\tau_{\mathrm{p}}$ is the transmitter pulse length, and $\tau_{\mathrm{T} \times \mathrm{R} \times \mathrm{Guard}}$ and $\tau_{\mathrm{RxT} T \mathrm{Guard}}$ are the guard times, respectively, between $\mathrm{Tx}$ and $\mathrm{Rx}$ and vice versa. The available sampling time is limited by hardware constraints. Examples of such constraints are the commanding transfer time between the SAR controller unit and the designated control module in the RF unit, activation time of the RF pulse gate for the Standardized Modular Transmit/ReceiveModule (SMTR-Module) in the RF unit, and delay of the timing signal from the controller to the SMTR-Module. The hardware constraints are considered by setting the guard times.

An echo resulting from nadir exists for every transmitted pulse, so every echo occurs at time $\tau_{\text {nadir }}$, which is defined as follows.

$$
\tau_{\text {nadir }}=\frac{2 h}{c}
$$

Here, $h$ is the altitude over the nadir point. Like the transmitter pulse eclipsing, PRFs should be determined to avoid coincidence between nadir echoes and the scene echo, so the following inequality should be satisfied.

$\frac{M-1}{\tau_{\text {near }}-\tau_{\mathrm{p}}-\tau_{\text {nadir }}-\tau_{\text {TxRxGuard }}}<P R F<\frac{M}{\tau_{\text {far }}+\tau_{\mathrm{p}}+\tau_{\text {nadir }}+\tau_{\text {RxTxGuard }}}(M=1,2,3, \ldots)$

PRF depends on the return times, $\tau_{\text {near }}$ and $\tau_{\mathrm{far}}$, which correspond to time at each slant, respectively. Also, it depends on a length of the transmitting pulse, $\tau_{p}$, and the altitude of the radar over the nadir point. The swath design and the PRF determination 
should be validated using a PRF diamond diagram. As an example, Figure 8 shows the PRF determination for SW\#5. The optimum PRF is determined by comparing Figure 8a with Figure 3: the PRF zone is determined to satisfy the requirement on the RAR and AAR performances. As shown in Figure 8b, this optimization procedure results in PRF at $8956.5 \mathrm{~Hz}$.

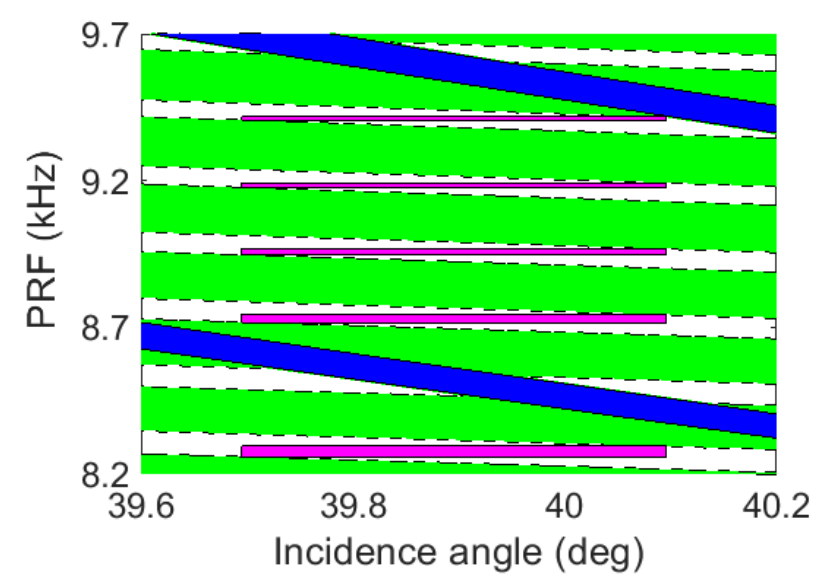

(a)

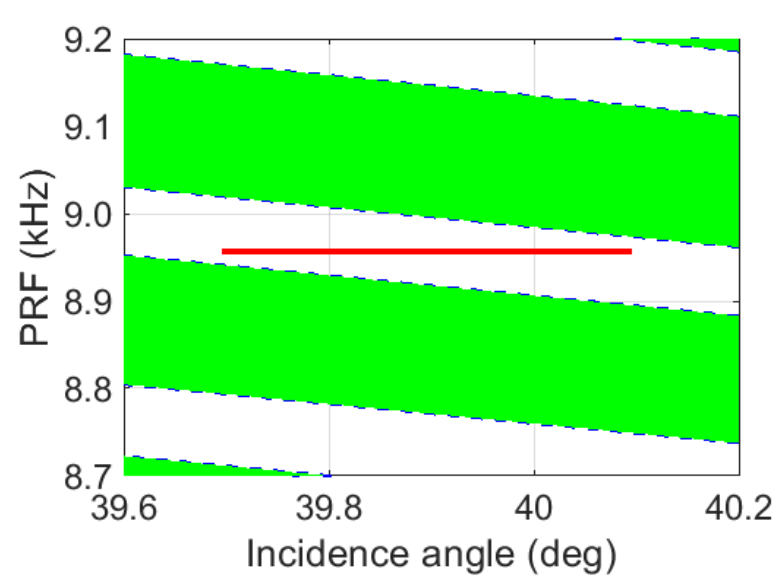

(b)

Figure 8. PRF determination for SW\#5: (a) Area in magenta: allocable PRF zones, area in green: interferences from transmitting returns, area in blue: nadir returns; (b) area in red: determined PRF at $8956.5 \mathrm{~Hz}$.

\subsection{Design Results on Nominal SAR Operational Parameters}

The procedure described in the previous sections are used to calculate the SAR operational parameters for each swath. Table 3 shows the nominal SAR operational parameters that are calculated for each swath. It also shows performances that are predicted for the sliding spotlight mode. In the parameter calculation and the performance prediction, the nominal altitude, incidence angle range, and swath width are $520 \mathrm{~km}, 20-55^{\circ}$, and $5.3 \mathrm{~km}$, respectively. Also, the Tx duty ratio, hamming window coefficient $(\alpha)$ in range and azimuth are $30 \%, 0.77$ and 0.54 , respectively.

Table 3. Nominal SAR operational parameters and corresponding performances at nominal altitude.

\begin{tabular}{ccccccccc}
\hline Parameter & SW\#1 & SW\#2 & SW\#3 & SW\#4 & SW\#5 & SW\#6 & SW\#7 & SW\#8 \\
\hline Incidence angle at center $\left(^{\circ}\right)$ & 20.03 & 25.14 & 29.91 & 35.16 & 39.90 & 45.14 & 50.00 & 54.93 \\
PRF (Hz) & 11839.6 & 8549.9 & 11755.5 & 11874.6 & 8956.5 & 6849.7 & 5742.9 & 4691.9 \\
Tx pulse length (us) & 25.33 & 35.09 & 25.52 & 25.26 & 33.49 & 43.79 & 52.24 & 63.93 \\
Maximum azimuth steering angle $\left(^{\circ}\right)$ & 1.65 & 1.63 & 1.60 & 1.56 & 1.51 & 1.47 & 1.42 & 1.43 \\
Image acquisition time (s) & 5.96 & 6.07 & 6.19 & 6.37 & 6.56 & 6.84 & 7.16 & 7.78 \\
NESZ (dB) & -26.41 & -25.57 & -24.69 & -24.35 & -23.77 & -22.98 & -21.96 & -20.96 \\
RAR (dB) & -31.42 & -34.87 & -26.06 & -24.64 & -28.63 & -28.61 & -25.93 & -21.25 \\
AAR (dB) & -38.49 & -33.16 & -38.19 & -38.40 & -32.60 & -27.85 & -26.36 & -24.50 \\
\hline
\end{tabular}

Figure 9 shows the SAR performances at the nominal altitude. All the performances satisfy the requirements in Table 1 for the whole range of the incidence angle. 


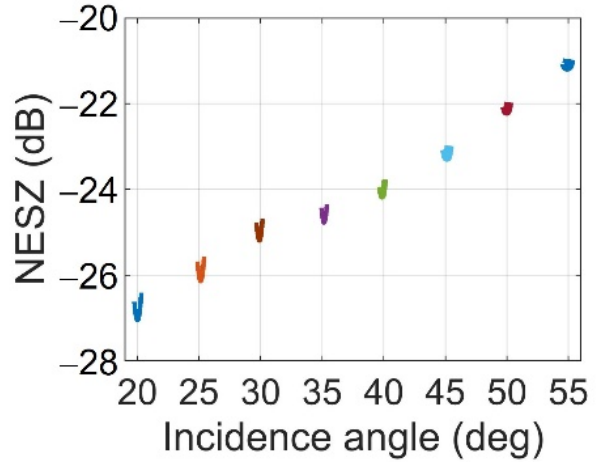

(a)

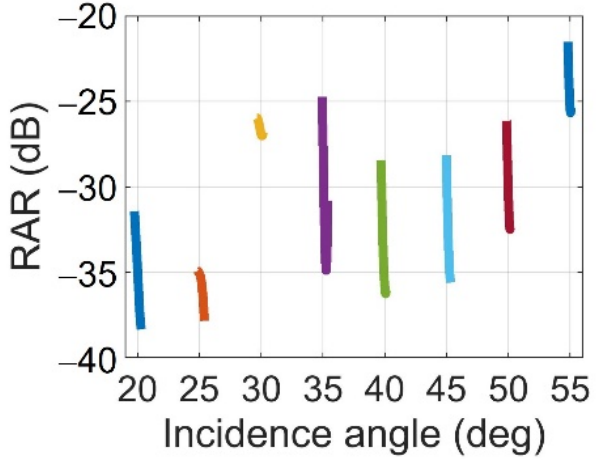

(b)

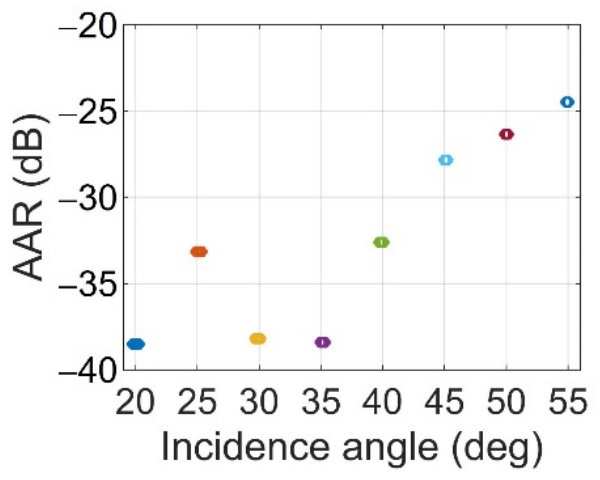

(c)

Figure 9. SAR performances at nominal altitude: (a) NESZ; (b) RAR; (c) AAR.

\section{Optimizing In-Orbit SAR Operational Parameters for Altitude Variation}

Figure 10 shows the flowchart to optimize the SAR operational parameters when a SAR satellite is in orbit. At first, the target is defined by a user for image acquisition. In this process, the swath numbers corresponding to the target are determined to cover the incidence angle range at the predicted altitude. The nominal SAR operational parameters are extracted from the database, which includes values presented in Table 3. The extracted parameters are as follows: (1) antenna parameters such as a RF center frequency, an antenna $\mathrm{Tx} / \mathrm{Rx}$ amplitude/phase excitation value, and an antenna beam pointing angle, (2) transceiver parameters such as Rx gain control and RF bandwidth, (3) SAR controller parameters such as a chirp rate, a Rx sampling frequency, a Block Adaptive Quantization (BAQ) compression ratio, and a Rx decimation ratio. As shown in Figure 10, those parameters are then used to recalculate the in-orbit SAR operational parameters for the predicted altitude. The following sections explain the processes for the target definition and the PRF optimization and finally, the optimization results. 


\section{Mission Planning}

- Target Definition

- Orbit prediction result (e.g., predicted satellite altitude)

- SAR operational mode (e.g., sliding spotlight, staring spotlight)

Swath number

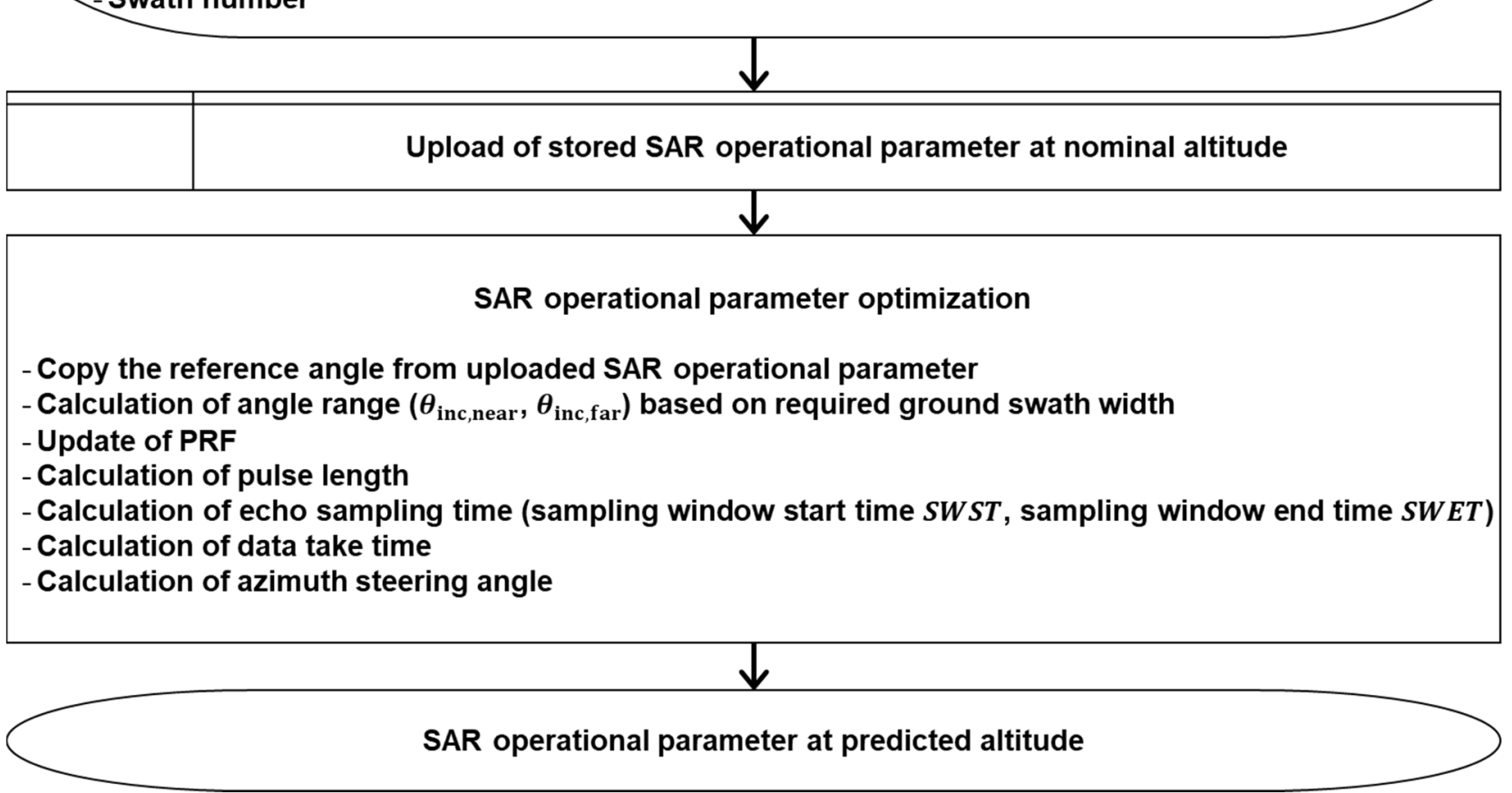

Figure 10. Flow chart for generating in-orbit SAR operational parameters.

\subsection{Definition of Swath Angle for Image Acquisition}

As a reference angle, an angle of the swath within a near to far range should be defined to generate the access angle range for a SAR system. The reference angle affects the SAR operational parameter generation and the antenna pattern generation, and finally, determines the SAR performance. In case of the previous heritage KOMPSAT-5, the reference angle was defined as the incidence angle corresponding to the middle of the swaths. The angle range for image acquisition was calculated based on the required ground swath width and incidence angle. The center of the angle range was set to the same as the center between the near and far incidence angles of all the swaths at the nominal altitude. This angle was constant regardless of the altitude variation. The near and far incidence angles were then calculated to comply with the required ground swath width. For KOMPSAT-6, however, the reference angle is defined in a straightforward way. The look angle can be used as the reference angle to calculate the angle range of the near and far swaths at the predicted altitude.

Image acquisition is strongly affected by which one between the incidence and look angles is used for the reference angle. During the in-orbit operation, beam pointing of the SAR antenna is dependent on the satellite altitude where the image acquisition is executed. Therefore, the deviation of the beam pointing should be reflected to the swath design considering the altitude variation. In the previous KOMPSAT-5, the incidence angle was used for the reference angle. Figure 11 shows examples of a SAR image that was acquired during the early operation phase of KOMPSAT-5. Each figure was acquired at a different altitude. As shown in Figure 11, the acquired images have an asymmetric property even though the image was acquired for Greenland, in which is supposed to produce an image having uniform intensity. This is because the incidence angle at the nominal altitude was used for the one at the predicted altitude without considering the altitude variation. The incidence angle was then used to acquire an image even though the actual altitude 
was changed to 566.285 and $569.907 \mathrm{~km}$, respectively, from $550 \mathrm{~km}$, which is the nominal altitude of KOMPSAT-5. The altitude variation causes the beam pointing error of about $0.07^{\circ}$ in the antenna pattern.

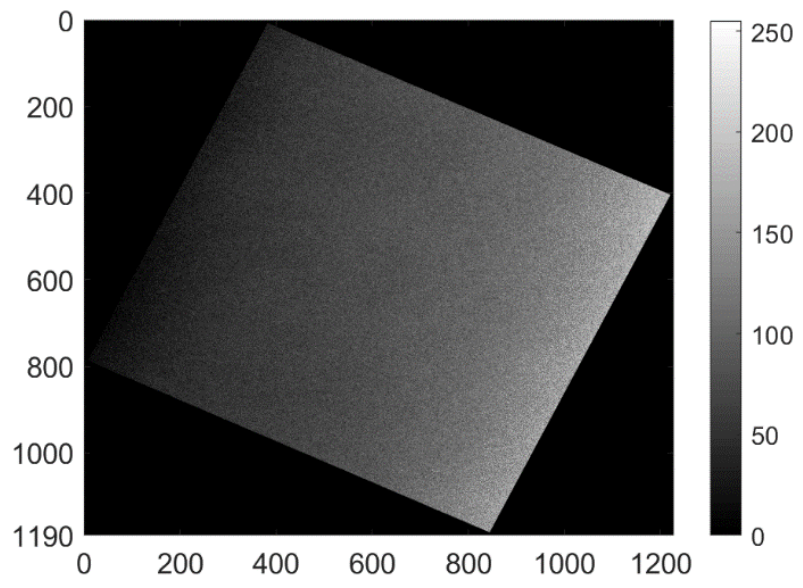

(a)

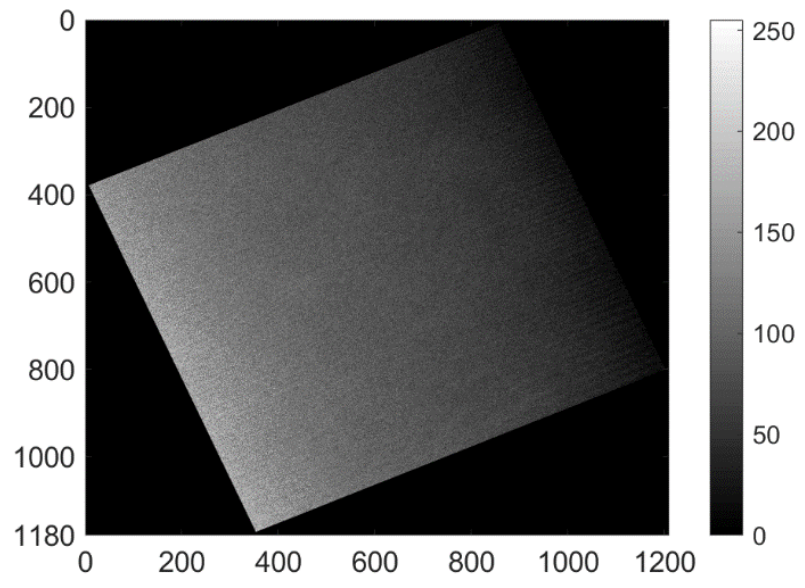

(b)

Figure 11. Examples of SAR image: (a) actual altitude is $566.285 \mathrm{~km}$ in ascending orbit; (b) actual altitude is $569.907 \mathrm{~km}$ in descending orbit.

Figure 12 shows the look angle variation at near, center and far position, respectively, across the swaths when the altitude changes between 509 and $540 \mathrm{~km}$. Figure 12a shows the look angle variation when the incidence angle is used for the reference angle without any correction as done in KOMPSAT-5. When the altitude changes between the minimum and maximum ones, the look angle also changes significantly for all the positions of the swaths. As shown in Figure 12b, however, the change in the look angle becomes minimal when the look angle is used for the reference angle. The same altitude variation changes the look angle much less than before. As a result, utilization of the look angle results in a smaller change in the beam error value for each altitude.

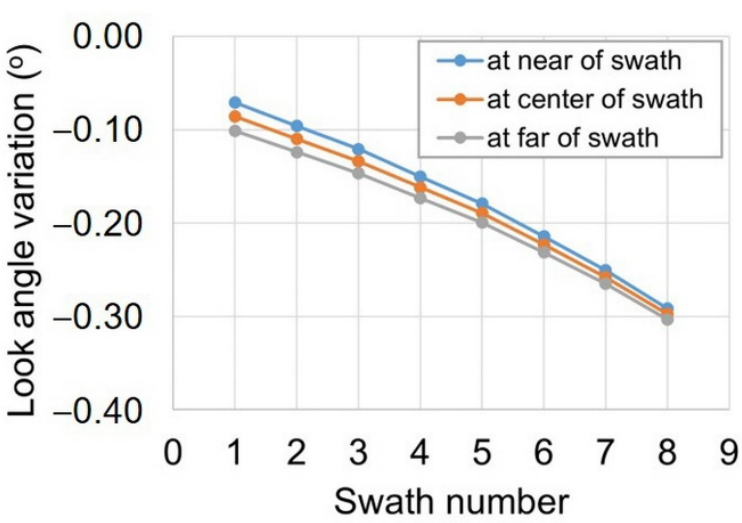

(a)

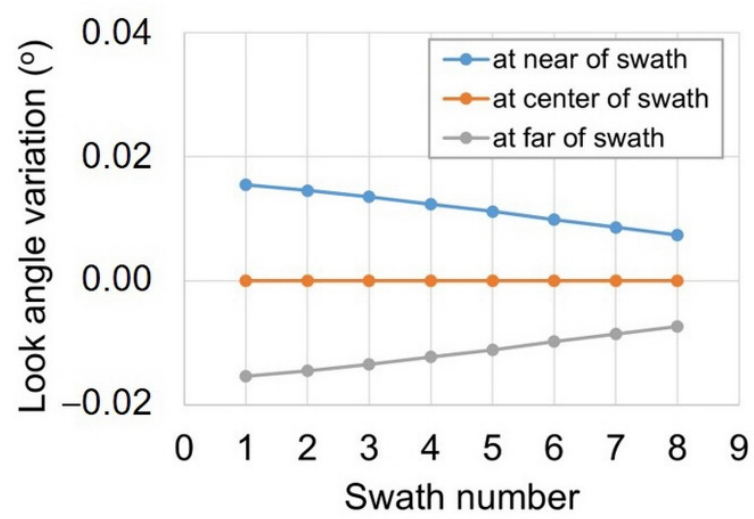

(b)

Figure 12. Look angle variation between minimum and maximum altitude when (a) incidence angle; (b) look angle at center of swath, respectively, are used for the reference angle.

For SAR application, an antenna pattern affecting the RAR performance is designed based on the look angle. Figure 13 compares the RAR performances when the incidence and the look angles, respectively, are used for the reference angle in the two swaths. The RAR performance significantly changes according to the altitude variation. The RAR performance is much better when the reference angle is based on the look angle instead of 
the incidence angle. As shown in Figure 13a,c, it is difficult to obtain good performance at the maximum altitude when the incidence angle is used for the reference angle. As shown in Figure 13b,d, however, the RAR performance becomes constant irrespective of the altitude variation when the look angle is used for the reference angle. In this case, the performance curve is generally shifted to the left side for the higher altitude.

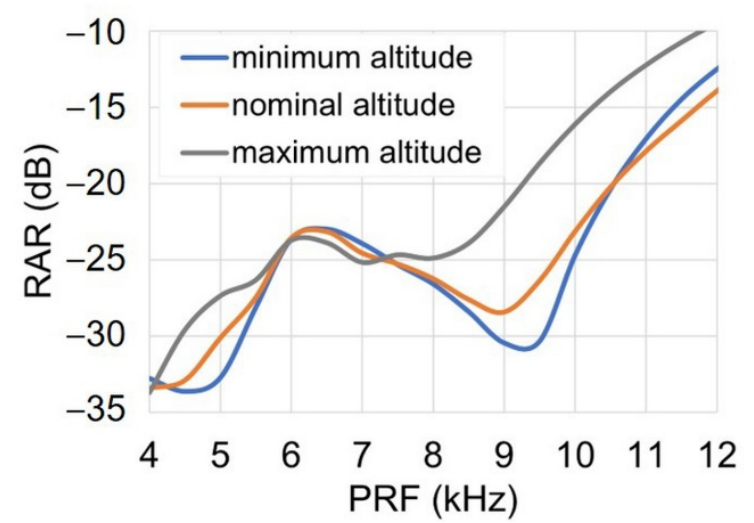

(a)

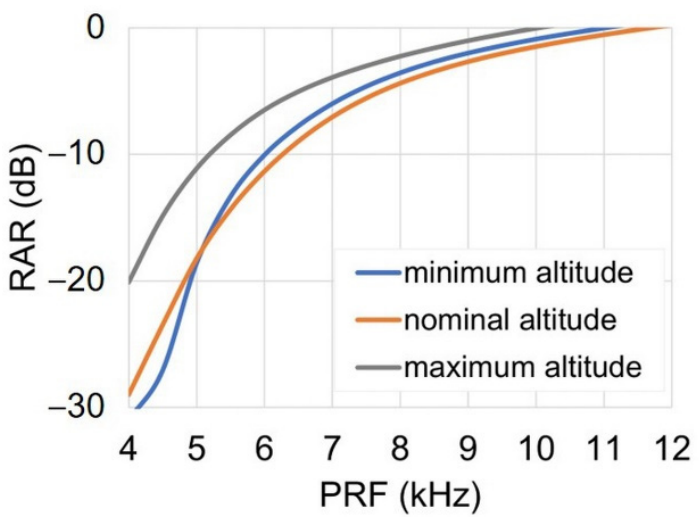

(c)

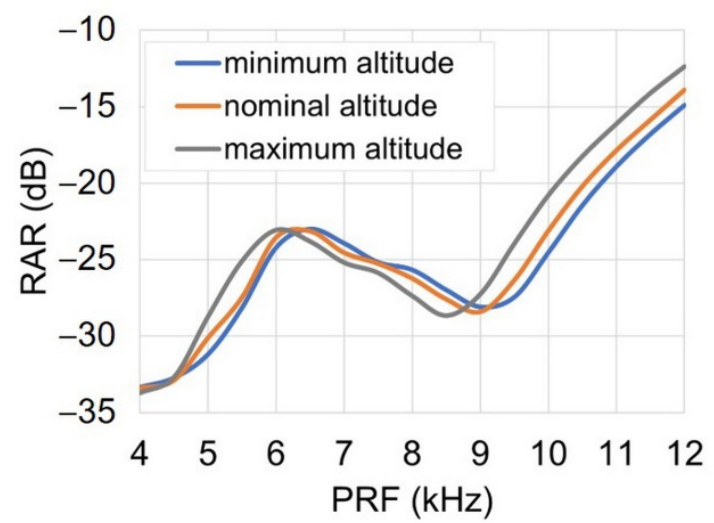

(b)

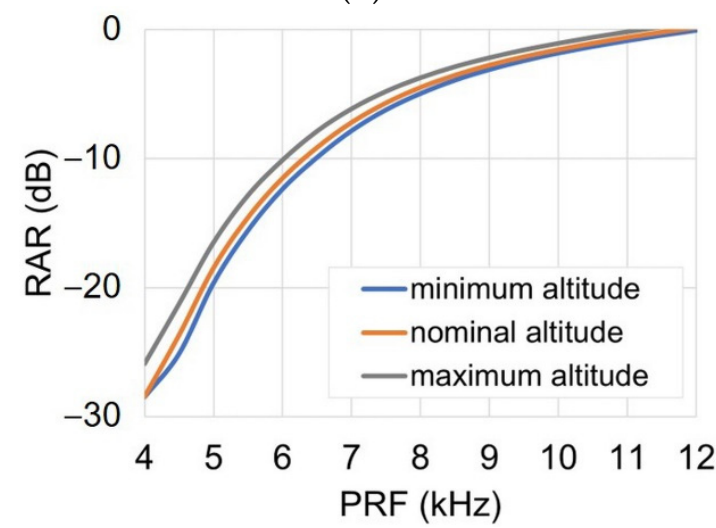

(d)

Figure 13. Examples of RAR performances for altitude variation, respectively, when (a) incidence angle and (b) look angle at center of swath are used for the reference angle in SW\#5, and when (c) incidence angle and (d) look angle at center of swath are used for the reference angel in SW\#8.

\subsection{PRF Optimization}

After defining the swath for the image acquisition, PRF should be optimized considering the altitude variation. Three methods can be used for the PRF optimization. The first method is to select constant PRF neglecting the altitude variation. The second method, which was used for KOMPSAT-5, is to optimize PRF using the ratio between the nominal and predicted altitudes. The third method is a new one proposed in this paper. PRF is optimized using the traveling return time.

\subsubsection{Selection of Constant PRF}

This method uses the PRF diamond diagram shown in Figure 14. The final PRF is selected within the white clear regions in the PRF diamond diagram. In each subfigure of Figure 14, the green and blue areas represent $\mathrm{T} x$ and nadir interference regions, respectively. The two areas are drawn to be overlaid each other for three altitudes: the minimum, nominal, and maximum altitudes. The white area represents the allocable PRF range. As shown in Figure 14, the allocable range becomes narrower for the increasing incidence angle, and no allocable range exists for SW\#8. As a result, this method has limitation to be applied only for the lower incidence angle range. 


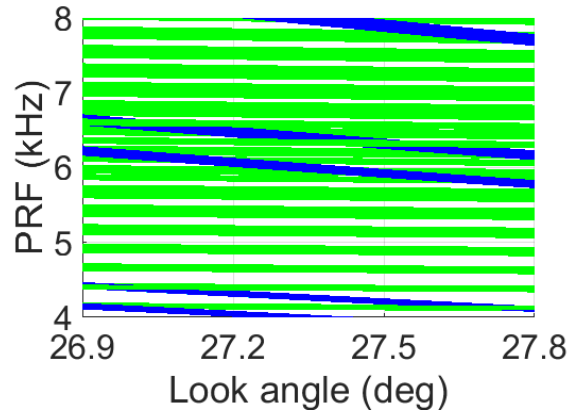

(a)

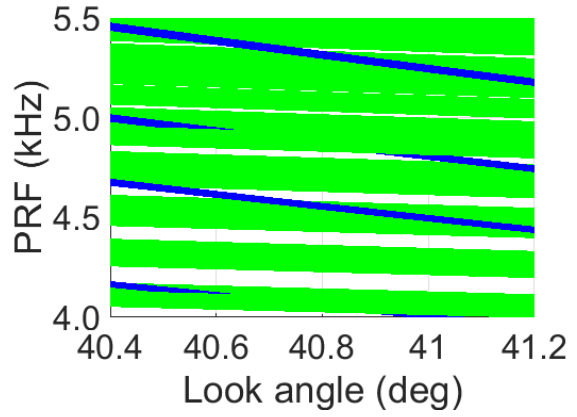

(b)

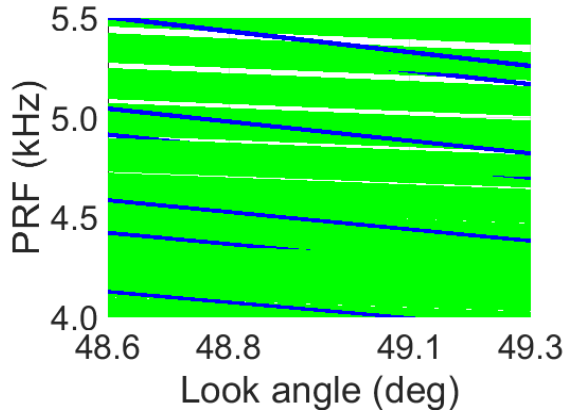

(c)

Figure 14. Available PRF range in PRF diamond diagram: (a) SW\#3; (b) SW\#6; (c) SW\#8.

If the altitude variation is small and the allocable PRF range exists simultaneously, an alternative method is to apply the constant RPF only for low look angles of Figure 14a. This method is easy to apply for the in-orbit operation, but the SAR performance is degraded as the actual altitude starts to deviate from the predicted altitude.

\subsubsection{PRF Optimization Using the Altitude Ratio}

In this approach, PRF is allowed to change according to the altitude variation to overcome the limitation of the constant PRF selection method. For this, PRF is optimized using the ratio between the nominal and predicted altitudes. As shown in Figure 13, the allocable PRF value decreases for the increasing altitude. Using such proportional relation between PRF and the altitude, the optimized PRF, $P R F_{\mathrm{opt}}$, is given as follows.

$$
P R F_{\mathrm{opt}}=\frac{H_{\mathrm{opt}}}{H_{\text {nominal }}} P R F_{\text {nominal }}
$$

Here, $H_{\text {opt }}$ is the predicted altitude. Also, $H_{\text {nominal }}$ and $P R F_{\text {nominal }}$ represent, respectively, the nominal satellite altitude and the PRF value at the nominal altitude, which are stored in the database. This method was used for KOMPSAT- 5 before this study.

\subsubsection{PRF Optimization Using Travelling Return Time}

PRF is related to the echo sampling time that is defined as the time interval between the sampling window start time (SWST) and sampling window end time (SWET). The travelling return time to the horizontal area is defined as Rank. SWST, SWET, and Rank are expressed, respectively, as follows [9].

$$
\begin{gathered}
S W S T=\frac{2 R_{\mathrm{s}, \text { near }}}{c}-\frac{\text { Rank }}{P R F} \\
S W E T=\frac{2 R_{\mathrm{s}, \mathrm{far}}}{c}-\frac{\text { Rank }}{P R F}+\tau_{\mathrm{p}} \\
\text { Rank }=f i x\left|\frac{2 R_{\mathrm{s}, \text { near }}}{c} \cdot P R F\right|
\end{gathered}
$$

Here, the function fix $(\cdot)$ means the decimal cut. The echo sampling time is a function

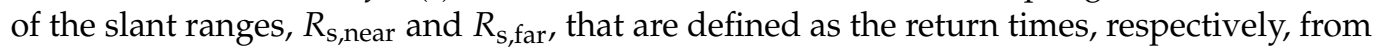
near incidence angle and far incidence angle of the swath. Using the guard times, $\tau_{\text {TxRxGuard }}$ and $\tau_{\text {RxTxGuard }}$ in Figure 7 , inequalities for SWST and SWET are expressed as follows.

$$
\begin{gathered}
S W S T \geq \tau_{\mathrm{p}}+\tau_{\text {TxRxGuard }} \\
S W E T \leq \frac{1}{P R F}-\tau_{\text {RxTxGuard }}
\end{gathered}
$$


The minimum PRF is calculated using Equations (7) and (10) as follows.

$$
P R F \geq \frac{T x_{\text {duty }}+\text { Rank }}{\frac{2 R_{\mathrm{s}, \text { near }}}{c}-\tau_{\text {TxRxGuard }}}
$$

Also, the maximum PRF is calculated using Equations (8) and (11) as follows.

$$
P R F \leq \frac{R a n k+1-T x_{\text {duty }}}{\frac{2 R_{\mathrm{s}, \mathrm{far}}}{c}+\tau_{\text {RxTxGuard }}}
$$

The allowable upper and lower limits of PRF are derived from the above the SWST and SWET inequalities.

$$
\frac{T x_{\text {duty }}+\text { Rank }}{\frac{2 R_{\mathrm{s}, \text { near }}}{c}-\tau_{\text {TxRxGuard }}} \leq P R F \leq \frac{\text { Rank }+1-T x_{\text {duty }}}{\frac{2 R_{\mathrm{s}, \text { far }}}{c}+\tau_{\text {RxTxGuard }}}
$$

The optimized PRF is given as an average of the PRF upper and lower limits in Equation (15).

$$
P R F_{\text {opt }}=\frac{1}{2}\left\{\frac{T x_{\text {duty }}+\text { Rank }}{\left(\tau_{\text {near }}-\tau_{\text {TxRxGuard }}\right)}+\frac{R a n k+1-T x_{\text {duty }}}{\left(\tau_{\text {far }}+\tau_{\text {RxTxGuard }}\right)}\right\}
$$

\subsubsection{PRF Optimization Results}

Using each of the three methods, PRFs are obtained and compared. Figure 15 shows examples for SW\#4 and SW\#8, respectively. In each subfigure, the solid lines in red represent the PRF values that are calculated at the nominal altitude with the altitude variation neglected. These constant PRFs are located within the Tx interference zone except for PRF of SW\#8 at the maximum altitude. The dotted lines in blue represent the PRF values that are calculated using the ratio between the nominal and predicted altitudes. PRFs are remarkably close to the Tx or nadir interference zone. If the actual altitude is deviated from the predicted one, PRF is highly probable to fall into the interference zone, and consequently, the range ambiguity performance is degraded. In Figure 15, the solid lines in purple represent the PRF values that are optimized using the travelling return time according to the proposed method. Contrary to other methods, PRFs are always located at the center of the clear zone.

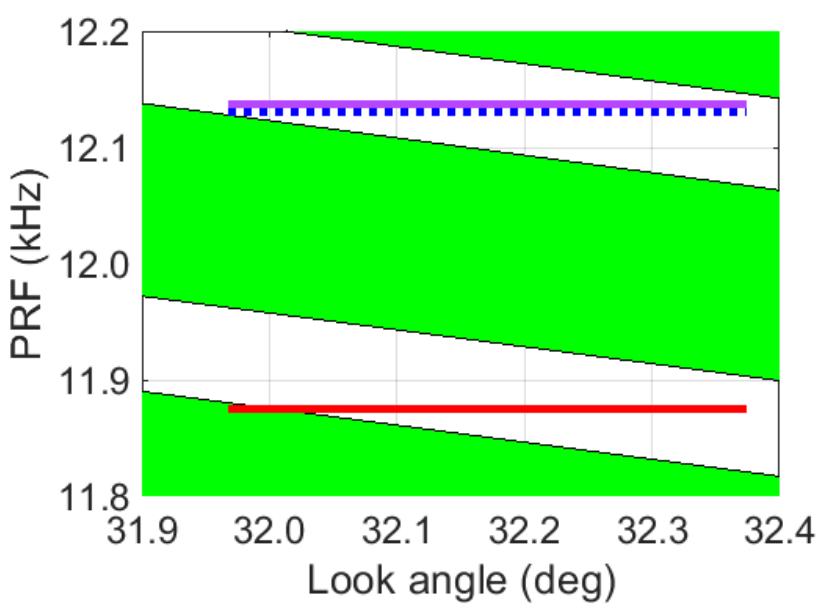

(a)

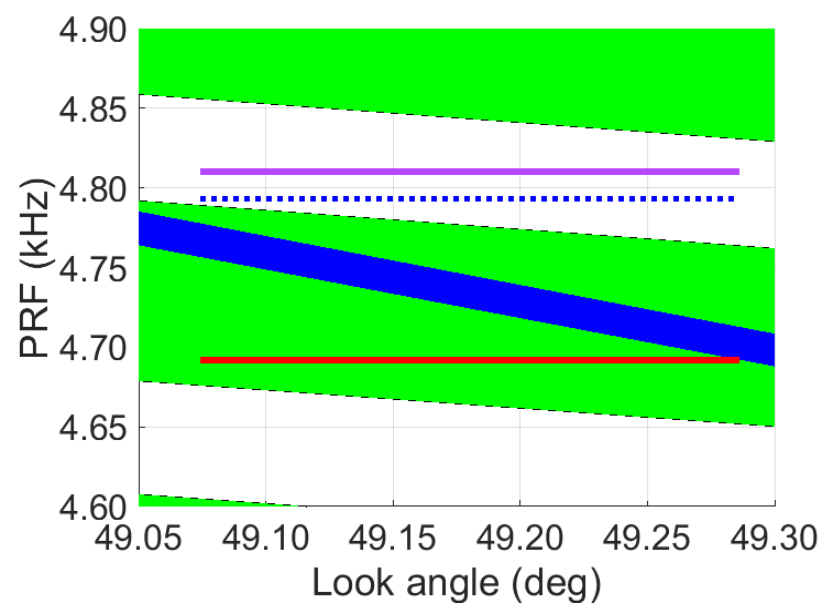

(b)

Figure 15. Cont. 


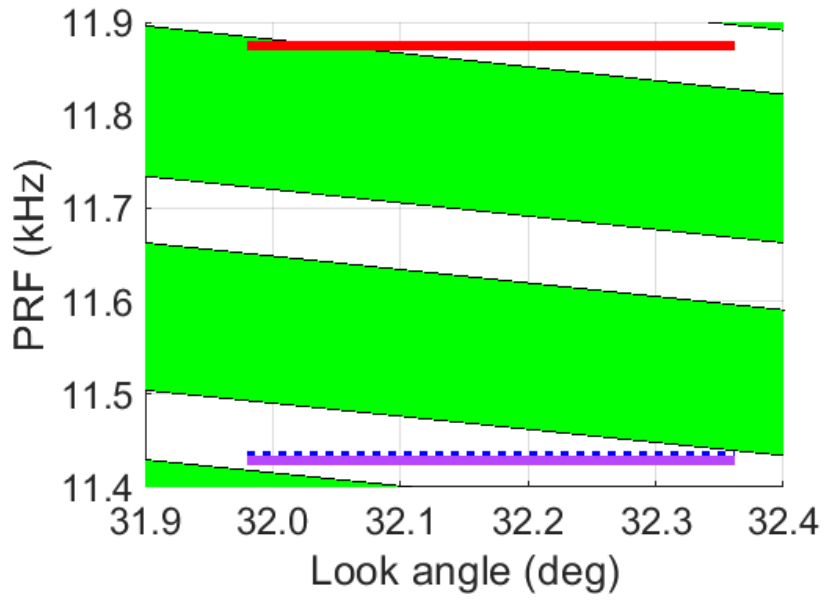

(c)

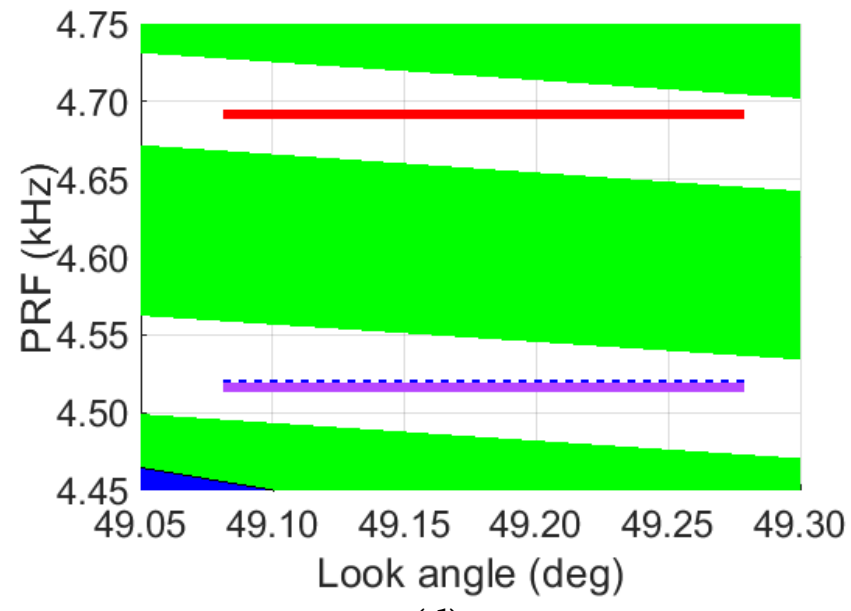

(d)

Figure 15. Example of PRF selection: (a) PRF of SW\#4 at the minimum altitude; (b) PRF of SW\#8 at the minimum altitude; (c) PRF of SW\#4 at the maximum altitude; (d) PRF of SW\#8 at the maximum altitude.

Figure 16 shows the PRF margins at the minimum and maximum altitudes, respectively. The upper margin is the distance to PRF at the maximum look angle from the Tx or nadir interferences. In similar, the lower margin is the distance to PRF at the minimum look angle from the Tx or nadir interference. As shown in Figure 16, the margins for PRF and the slant angle are different when PRF is optimized using the second method, i.e., calculated using the ratio between the nominal and predicted altitudes. Especially, the margins are ridiculously small for several swaths, so the PRF margin is reduced below $5 \mathrm{~Hz}$ while the slant range margin below $6 \mathrm{~m}$. The proposed method, however, results in the margins that are the same irrespective of the altitude variation. Also, no significant reduction in the PRF and slant range margins is observed.

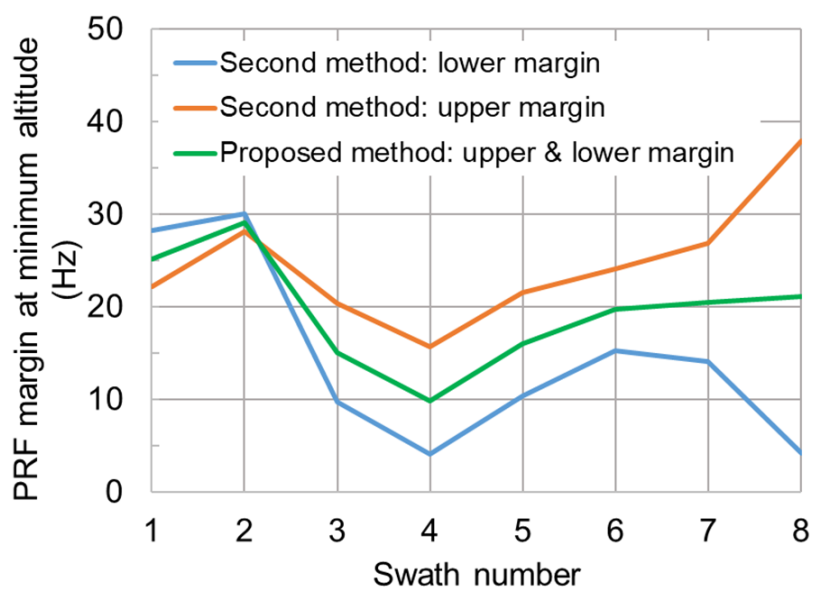

(a)

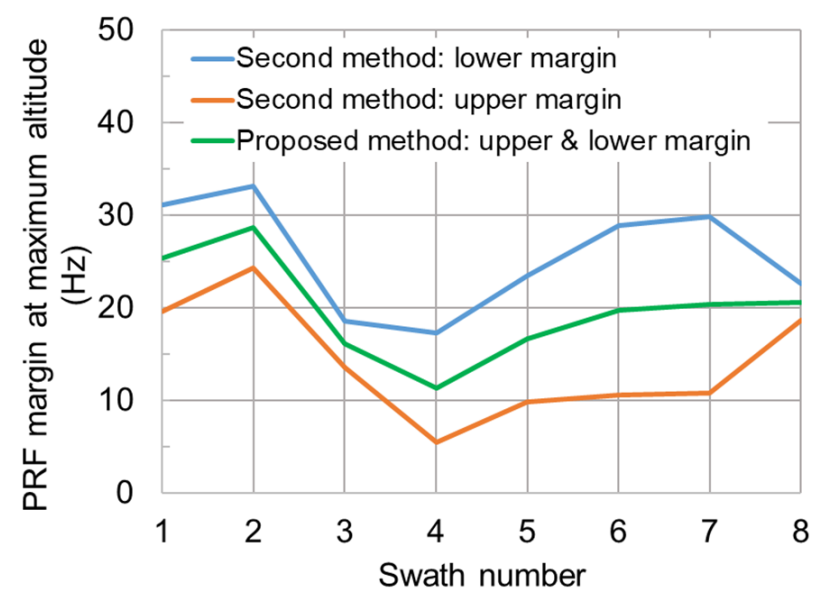

(b)

Figure 16. Cont. 


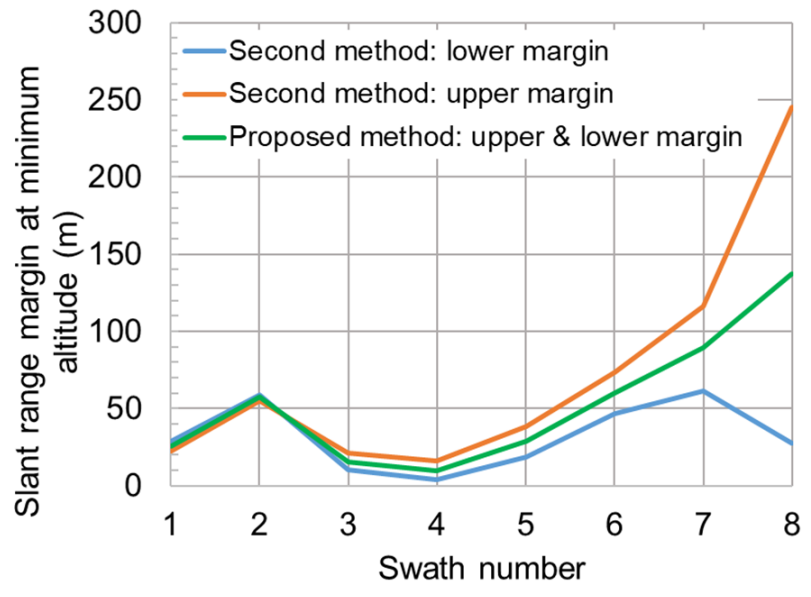

(c)

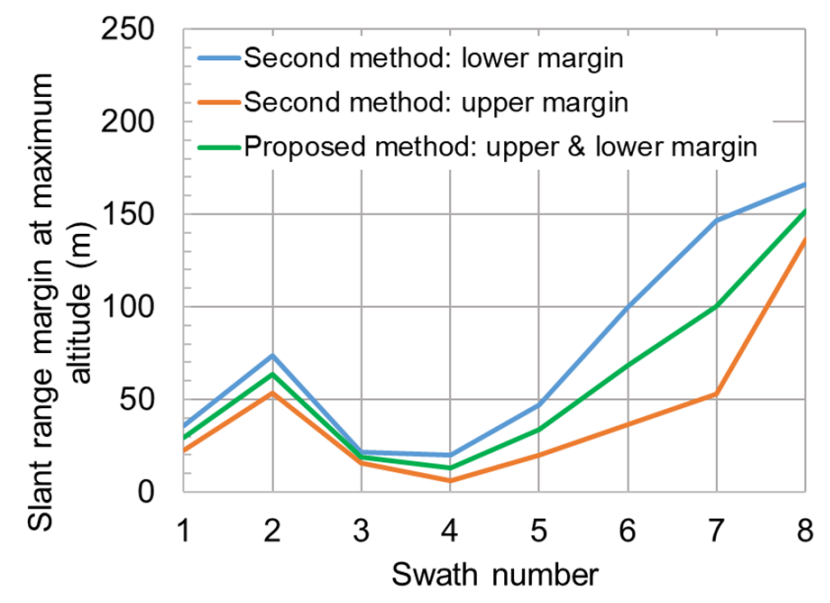

(d)

Figure 16. PRF and slant range margins according to altitude variation for: (a) PRF margin at the minimum altitude; (b) PRF margin at max. altitude; (c) slant range margin at the minimum altitude; (d) slant range margin at the maximum altitude.

\section{Results}

\subsection{Optimization Results for In-Orbit SAR Operational Paramters}

Tables 4 and 5 show the SAR operational parameters that have been optimized considering the altitude variation when a satellite is in orbit. The designed ground swath width is $5.3 \mathrm{~km}$. PRF has been optimized using proposed method while other parameters have been designed using the look angle as the reference angle. Due to the optimization procedure, the parameters related to the SAR instrument operation are different from those in Table 3.

Table 4. Optimized SAR operational parameters at minimum altitude.

\begin{tabular}{ccccccccc}
\hline Parameter & SW\#1 & SW\#2 & SW\#3 & SW\#4 & SW\#5 & SW\#6 & SW\#7 & SW\#8 \\
\hline Incidence angle at center $\left(^{\circ}\right)$ & 19.99 & 25.10 & 29.86 & 35.10 & 39.82 & 45.05 & 49.89 & 54.80 \\
PRF (Hz) & $12,092.43$ & 8733.80 & $12,014.83$ & $12,137.03$ & 9155.67 & 7002.14 & 5873.40 & 4810.17 \\
Tx pulse length (us) & 24.81 & 34.35 & 24.97 & 24.72 & 32.76 & 42.84 & 51.08 & 62.36 \\
Maximum azimuth steering angle $\left(^{\circ}\right)$ & 1.68 & 1.65 & 1.62 & 1.58 & 1.53 & 1.48 & 1.43 & 1.45 \\
Image acquisition time (s) & 5.90 & 6.00 & 6.12 & 6.29 & 6.48 & 6.75 & 7.06 & 7.67 \\
\hline
\end{tabular}

Table 5. Optimized SAR operational parameters at maximum altitude.

\begin{tabular}{ccccccccc}
\hline Parameter & SW\#1 & SW\#2 & SW\#3 & SW\#4 & SW\#5 & SW\#6 & SW\#7 & SW\#8 \\
\hline Incidence angle at center $\left(^{\circ}\right)$ & 20.09 & 25.22 & 30.01 & 35.28 & 40.03 & 45.31 & 50.20 & 55.17 \\
PRF (Hz) & $11,395.36$ & 8228.86 & $11,317.62$ & $11,428.90$ & 8618.00 & 6586.90 & 5520.74 & 4516.21 \\
Tx pulse length (us) & 26.32 & 36.46 & 26.51 & 26.25 & 34.81 & 45.54 & 54.33 & 66.42 \\
Maximum azimuth steering angle $\left(^{\circ}\right)$ & 1.62 & 1.59 & 1.56 & 1.52 & 1.48 & 1.43 & 1.39 & 1.40 \\
Image acquisition time (s) & 6.08 & 6.19 & 6.32 & 6.51 & 6.71 & 7.00 & 7.34 & 7.98 \\
\hline
\end{tabular}

Figure 17 shows the SAR performances: NESZ, RAR, and AAR for the altitude variation. In Figure 17, the blue and green lines represent the SAR performances at the minimum and maximum altitudes, respectively. Figure $17 \mathrm{a}-\mathrm{c}$ show NESZ, RAR, and AAR, respectively, for PRF that has been optimized using second method (i.e., calculated using the ratio between the nominal and predicted altitudes) while using the incidence angle as the reference angle. Each of NESZ and RAR significantly changes for the altitude variation. RAR at maximum altitude is out of the requirement. Figure $17 \mathrm{~d}-\mathrm{f}$ show NESZ, RAR, and AAR, respectively, for PRF that has been optimized using the proposed method while 
using the look angle as the reference angle. All the performances satisfy the requirements even for the altitude variation.

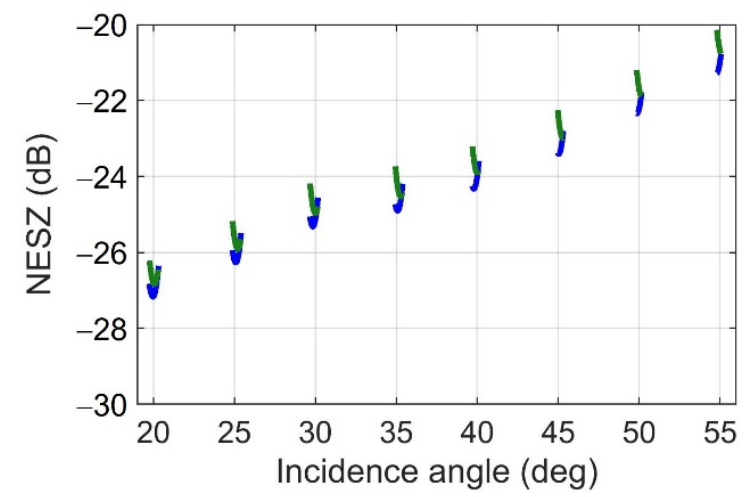

(a)

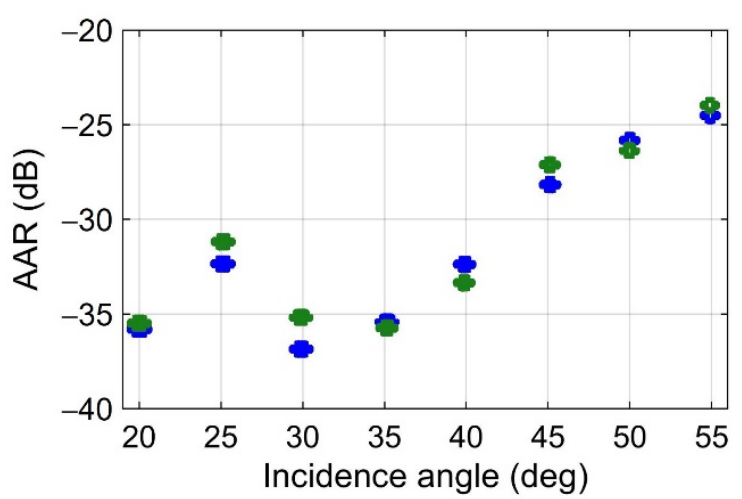

(c)

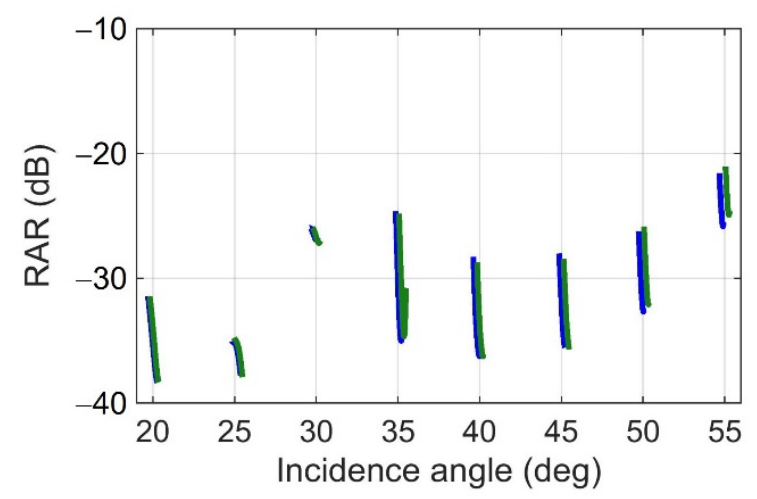

(e)

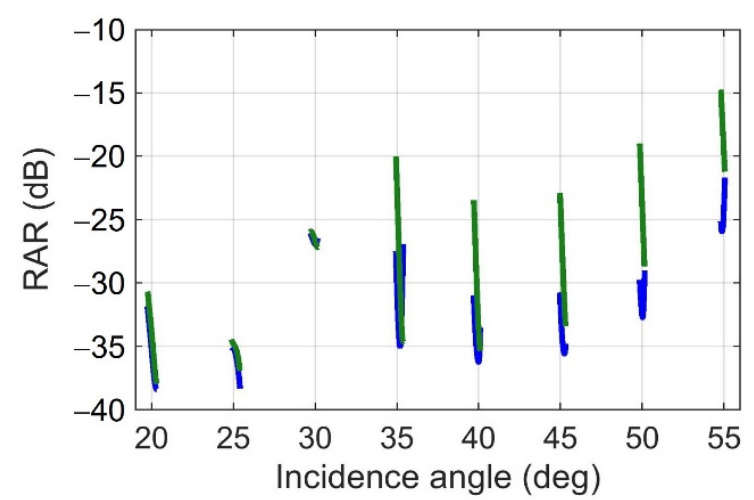

(b)

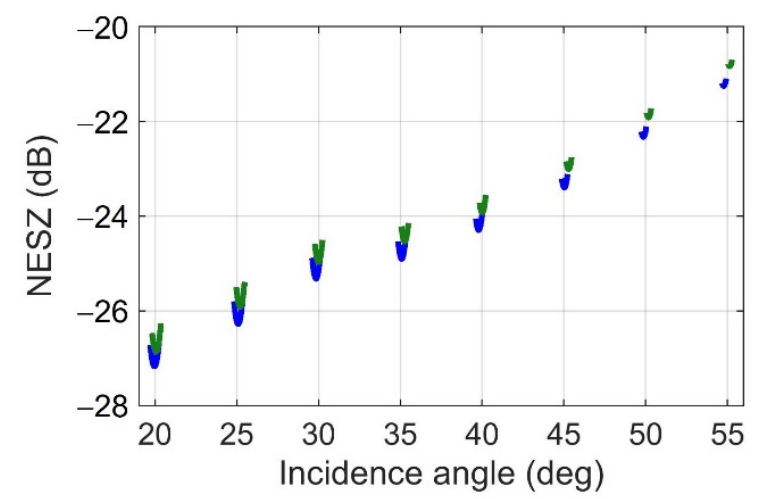

(d)

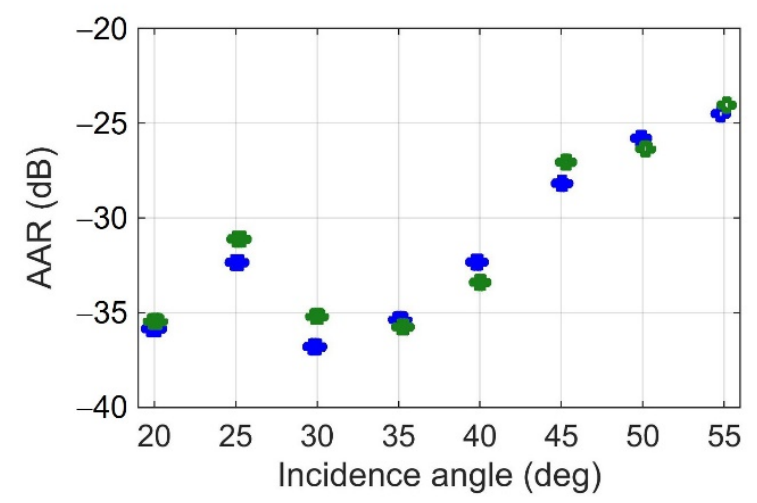

(f)

Figure 17. SAR Performances: (a) NESZ, (b) RAR, (c) AAR for PRF optimized with the second method while using the incidence angle as the reference one; (d) NESZ, (e) RAR, (f) AAR for PRF optimized with the proposed method while using the look angle as the reference one.

\subsection{On-Board PRF Optimization for Real-Time Operation}

The proposed method enables the on-board PRF optimization to be realized in a real time. In general, the SAR operational parameters are updated based on the altitude that is predicted at the ground segment. The updated table is then uploaded via the S-band uplink channel to the satellite prior to the image acquisition. The SAR controller uses the uploaded table for the image acquisition as preparing and managing the instrument timing for every pulse. In a conventional operation, the SAR operational parameters generated on-ground has HEX units. The SAR controller needs to convert the received parameters 
from HEX values to real ones and extract the real-time operational parameters based on mathematical equations stored in EEPROM. When the real-time operation is required due to urgent requests, such parameter update causes overload for the SAR controller because the time required for the update is relatively long. The update time, however, can be significantly reduced when PRF is optimized using the proposed method. This feature realizes the real-time operation. Figure 18 shows the procedure for the real-time operation. The SAR controller calculates the SAR operational parameters using the actual altitude that is transferred from the platform. Simultaneously, the SAR controller optimizes PRF using Equation (15). PRF is always located at the center of the clear zone even if the actual altitude is changed during the image acquisition or deviated from the predicted altitude. The parameter preparation procedure show in Figure 18 corresponds to the third procedure in Figure 10, SAR operational parameter optimization. For the parameter preparation time, the SAR controller just needs to implement the sub-routine that calculates and controls several operational parameters. Those parameters are optimized for the altitude variation. As a result, the proposed method does not overload the SAR controller even for real-time operation.

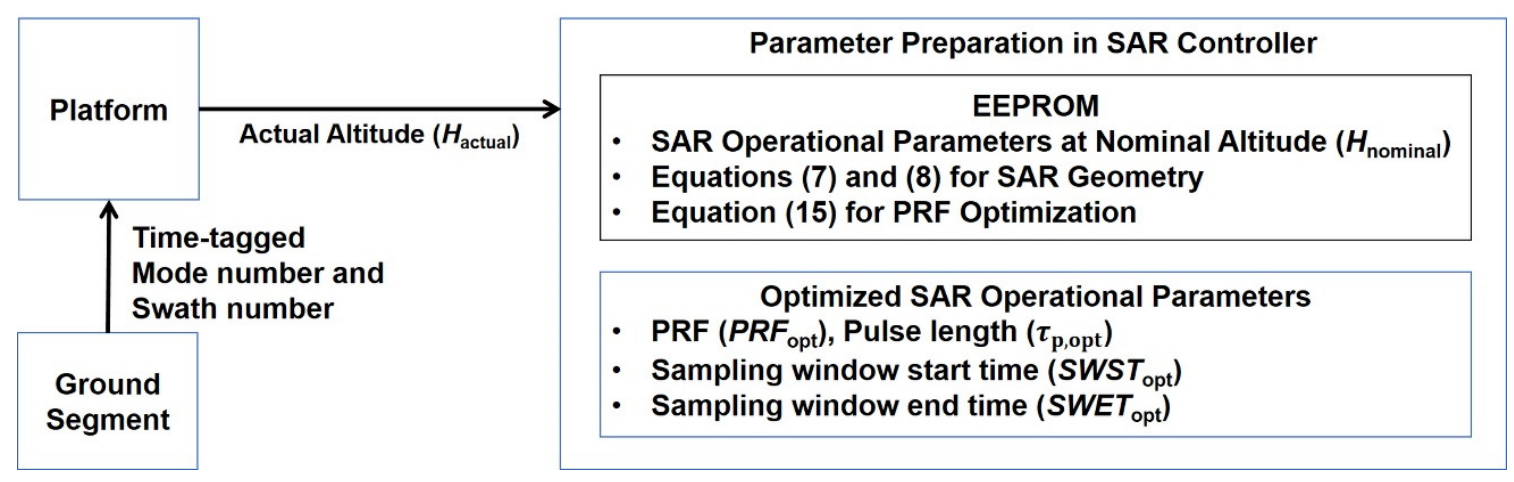

Figure 18. Procedure for real-time operation using proposed method.

\section{Discussions}

This paper proposes the method to design the SAR operational parameters for KOMPSAT-6. Among those parameters, PRF is an important one because it determines the ambiguity ratios including RAR and AAR. PRF is determined using a nominal altitude but should be optimized as the in-orbit altitude changes from the nominal altitude. The proposed method uses the traveling return time to optimize PRF. Utilization of the traveling return time enables PRF against the altitude variation to be corrected, and consequently, the altitude variation does not degrade the SAR performances including the PRF and slant range margins. In addition, the proposed method does not cause any overload for the SAR controller, so the on-board optimization for PRF makes it possible to implement real-time operation of a SAR satellite. Instead of the incidence angle, the proposed method uses the look angle for the reference angle to improve the SAR performances. Numerical simulation shows that the proposed method achieves higher SAR performances even for the altitude variation in comparison with the previous methods, which include the one used for KOMPSAT-5. The proposed method is based on the mathematical equations that have been derived in the theoretical way. Along with the numerical simulation, such fact indicates that the proposed method is general one applicable to other satellites.

As a further research, the impact of the real-world surface on SAR images will be studied by simulating the SAR imaging on real-world surface with various PRF selection approached. After launch of the KOMPSAT-6, the SAR performances will be evaluated using acquired SAR images, and the results will be presented in this journal. 


\section{Conclusions}

This paper proposed the optimization method for in-orbit operational parameters to maintain the SAR performances even in case of an altitude variation. The numerical simulation considering altitude variation is presented to validate the proposed optimization and the design methodologies.

Author Contributions: Conceptualization, J.-B.S. and S.-Y.H.; methodology, J.-B.S.; software, J.-B.S.; data curation, J.-B.S.; writing-original draft preparation, J.-B.S.; writing-review and editing, S.-Y.H.; supervision, S.-Y.H. All authors have read and agreed to the published version of the manuscript.

Funding: This research received no external funding.

Institutional Review Board Statement: Not applicable.

Informed Consent Statement: Not applicable.

Data Availability Statement: Not applicable.

Acknowledgments: The authors would like to thank the anonymous reviews for their comments to improve the paper quality.

Conflicts of Interest: The authors declare no conflict of interest.

\section{References}

1. Lee, S.-R.; Kim, J.-H.; Yoonk, J.-C. First SAR Satellite in Korea, KOMPSAT-5. Int. Conf. Space Aeronaut. Navig. Electron. (ICSANE) 2012, 112, 253-257.

2. Lee, S.-R. Overview of KOMPSAT-5 program, mission, and system. In Proceedings of the 2010 IEEE International Geoscience and Remote Sensing Symposium; Institute of Electrical and Electronics Engineers (IEEE), Honolulu, HI, USA, 25-30 July 2010; pp. 797-800.

3. Yoon, J.C.; Keum, J.H.; Shin, J.M.; Kim, J.H.; Lee, S.R.; Bauleo, A.; Farina, C.; Germani, C.; Mappini, M.; Venturini, R. KOMPSAT-5 SAR Design and Performance. In Proceedings of the 3rd International Asia-Pacific Conference on Synthetic Aperture Radar, APSAR, Seoul, Korea, 26-30 September 2011.

4. Bauelo, A.; Farina, C.; Germani, C.; Petrini, P.; Sirocchi, G.; Torini, A.; Won, Y.J.; Mun, H.Y.; Woo, S.H.; Kim, J.H.; et al. KOMPSAT-5 SAR P/L On the ground Verification Campaign. In Proceedings of the 3rd International Asia-Pacific Conference on Synthetic Aperture Radar, APSAR, Seoul, Korea, 26-30 September 2011.

5. Lee, S.-H.; Yoon, J.-C.; Kim, J.-H. KOMPSAT-6 Mission, Operation Concept, and System Design. In Proceedings of the EUSAR 2016: 11th European Conference on Synthetic Aperture Radar, Hamburg, Germany, 6-9 June 2016; pp. 161-164.

6. Hwang, Y.C.; Nam, C.H.; Pak, U.Y.; Kim, S.Y.; Lee, J.H. KOMPSAT-6 SAR Payload System Design. In Proceedings of the EUSAR 2016: 11th European Conference on Synthetic Aperture Radar, Hamburg, Germany, 6-9 June 2016; pp. 165-167.

7. Kang, C.; Jung, O.; Oh, T.; Yang, D.; Jeun, G. Operation concept of KOMPSAT-6 ground segment. In Proceedings of the EUSAR 2016: 11th European Conference on Synthetic Aperture Radar, Hamburg, Germany, 6-9 June 2016; pp. 168-173.

8. Kim, S.Y.; Myung, N.H.; Kang, M.J. Antenna Mask Design for SAR Performance Optimization. IEEE Geosci. Remote. Sens. Lett. 2009, 6, 443-447. [CrossRef]

9. Curlander, J.C.; McDonough, R.N. SAR Flight System. Synthetic Aperture Radar Systems and Signal Processing; Kong, J.A., Ed.; John Wiley \& Sons, Inc.: New York, NY, USA, 1991; pp. 294-308. 